\title{
Dwell time controllers for stochastic systems with switching Markov chain
}

\author{
S. Battilotti, A. De Santis ${ }^{\text {a }}$ \\ a Dip. di Informatica e Sistemistica, Università di Roma "La Sapienza", Via Eudossiana 18, 00184 Rome, Italy
}

\begin{abstract}
We study the problem of feedback stabilization of a family of nonlinear stochastic systems with switching mechanism modeled by a Markov chain. We introduce a novel notion of stability under switching, which guarantees a given probability that the trajectories of the system hit some target set in finite time and remaining thereinafter. Our main contribution is to prove that if the expectation of the time between two consecutive switching (dwell time) is "sufficiently large" then the system is stable under switching with guaranteed probability. We illustrate this methodology by constructing measurement feedback controllers for a wide class of stochastic nonlinear systems.
\end{abstract}

Key words: Stochastic systems, switching systems, measurement feedback.

\section{Introduction}

Robust control is an efficient design tool when the system can be characterized as a nominal model plus uncertainty with known bounds ([1]). These bounds represent a worst-case situation in which the controller is designed. Another case is the one in which the system switches within a finite number of models due to the presence of noise or uncertainty ([7]). The control law, known as switching control, consists of a family of controllers which stabilize each model and a supervision logic which selects at each switching time the controller to be employed in the control loop. The number of switching is assumed infinite since otherwise the stabilization problem would be trivial. It is known that a not suitable timing of the controllers may lead to a closed-loop system state diverging to infinity. For linear systems, it is also known that if the time bewteen each switching, usually known as dwell-time, is not less than some constant number, stability of the closed-loop system is guaranteed ([7]). Recently, a time-varying or state-dependent dwell-time has been introduced to design a switching controller for some classes of iISS ([8]) and ISS ([9]) nonlinear systems. The supervision logic selects from time to time the controller which stabilizes the system as long as the dwell-time is larger than some amount of time, which depends on the initial value of the state when the switching occurs.

A more complex situation is the one in which the switching mechanism is modeled as a Markov chain or even in which a noise, modeled as a Wiener process, affects each system of a given family. This is a very realistic situation when abrupt changes in the parameter structure occur due to component failures or repairs, environmental disturbances or changes in the system interconnections. A first contribution is given for a family of delayed linear systems in [10], where the technical conditions for achieving exponential stability in the mean square are formulated. The analysis is led through a Lyapunov-based approach, in which a Lyapunov function $V_{i}$ is available for each system $i \in I$, and the effect of the Markovian chain is taken into account by adding a "weighted average" of the Lyapunov functions $V_{j}, j \in I$, in the infinitesimal generator $L$ acting on $V_{i}$, the weights depending on the transition probabilities of the Markov chain.

In this paper, we want to extend the results of [10] in several directions. First of all, we consider a family of nonlinear systems, each consisting of a nominal system plus nonlinear stochastic term in which the noise is modeled as a Wiener process. As in [10] the switching mechanism is modeled as a Markov chain. We assume that a Lyapunov function $V_{i}$ and measurement feedback controller $C_{i}$ are available for each system $i$ of the family and the controller $C_{i}$ stabilizes the system $i$ in probability with guaranteed region of attraction and target set in the sense of [1]. Given numbers $\alpha_{i}, \beta_{i} \in[0,1)$ and a pair of compact sets $T_{i} \subset R_{i}$, containing the origin, the trajectories of the closed-loop system with initial condition in the region of attraction $R_{i}$ remain inside some larger compact set, eventually enter any given neighbourhood 
of the target set $T_{i}$ in finite time and remain thereinafter, all with probability at least $\left(1-\alpha_{i}\right)\left(1-\beta_{i}\right)$. The numbers $\alpha_{i}$ and $\beta_{i}$ can be considered as risk margins: the first one quantifies the risk of leaving the region of attraction rather than getting close to the target, while the second one gives a risk margin for remaining close to the target.

We also assume that for each level set $D_{i}$ of $V_{i}$ there exists a level set $D_{i+1}$ of $V_{i+1}$ contained in $D_{i}$. This nesting property was already used for the control of hybrid systems in [11]. The key idea is that the system trajectory dwells a sufficiently large amount of time in $D_{i}$ before the next switching occurs so that in the meanwhile it has approached the set $D_{i+1}$. If the sequence of sets $\left\{D_{i}\right\}$ is definitely contained or "close" to the target, then the system trajectory approaches the target set. We propose a switching strategy which consists of selecting at each switching time $i$ the controller $C_{i}$. To this aim, we introduce a notion of stabilization of the switching system (stabilization under switching with guaranteed probability: definition 2), which ensures a given probability of the system trajectories hitting $D_{i+1}$ conditionally to the event $\Xi_{i}$ of dwelling in $D_{i}$. Our main contribution is to prove that if the conditional expectation to the event $\Xi_{i}$ of the dwell-time is "sufficiently large" then the switching system is stabilizable under switching with guaranteed probability (theorem 3). As an application of our design tool, we consider a family of nonlinear stochastic systems and show in detail how to construct a switching controller using theorem 3. Moreover, our result is weaker even under the same assumptions of [10], since stability in probability is implied by mean square stability.

\section{Notations}

We give some notations extensively used throughout the paper.

- if $\|v\|$ denotes the $2-$ norm of any given vector $v$, by $\|A\|$ we denote the induced $2-$ norm of any given matrix $A$; by $\|v\|_{A}$ we denote the $A$-norm of $v$, i.e. $\|v\|_{A}=\sqrt{v^{T} A v}$; let col $\left(v_{1}, \ldots, v_{n}\right)$ be the column vector with $i$-th entry equal to $v_{i}$. Moreover, $\lambda_{\min }(A)$ and $\lambda_{\max }(A)$ denote the minimal and, respectively, maximal eigenvalues of a given square symmetric matrix $A$.

- by $S P^{n}$ (resp. $S N^{n}$ ) we denote the set of $n \times n$ positive (resp. negative) definite symmetric matrices; by $S S P^{n}$ we denote the set of $n \times n$ positive semidefinite symmetric matrices; $\mathbb{R}^{+}$denotes the set of positive real numbers and $\mathbb{R}^{\geq}$the set of nonnegative real numbers;

- for any vector-valued function $\eta: \mathbb{R}^{s} \rightarrow \mathbb{R}^{r}$, we denote by $\eta_{i}$ (or $[\eta]_{i}$ ) its $i$-th component. A continuous function $\alpha: \mathbb{R}^{\geq} \rightarrow \mathbb{R}^{\geq}$is said to be of class $K$ (or $\alpha \in K)$ if $\alpha(0)=0$ and it is increasing. For any smooth function $f: \mathbb{R}^{n} \rightarrow \mathbb{R}, s \mapsto f(s)$, and $\lambda: \mathbb{R}^{l} \rightarrow \mathbb{R}^{n}$, $r \mapsto \lambda(r)$, we denote by $\nabla_{s} f(s)$ the gradient of $f(s)$, by $\nabla_{s s}^{2} f(s)$ the Hessian of $f(s)$ and $\left.\nabla_{s} f\right|_{\lambda(r)}$ the gradient of $f(s)$ evaluated for $s=\lambda(r)$.

- for any given set $S$, we denote by $\operatorname{clos}(S)$ its closure, by $\partial S$ its boundary and by int $(S)$ its interior; moreover, given $\delta>0$ and a set $S$, by $\delta$-neighbourhood of $S$ we denote the set $S_{\delta}=\left\{z: \inf _{y \in S}\|z-y\|<\delta\right\}$. Moreover, by $R \backslash S$ denotes the the elements of $R$ which are not in $S$.

- $\boldsymbol{P r}\{\cdot\}$ denotes the probability measure, $\mathbf{E}\{\cdot\}$ denotes the expectation and $\mathbf{E}\{\cdot \mid \cdot\}$ the conditional expectation.

\section{Stability in probability with guaranteed re- gion of attraction and target set}

In this section we want to review some previous results on the stabilization for the following class of stochastic systems

$d x(t)=(A x(t)+B u(t)) d t+H(x(t)) d w$
$d y(t)=C x(t) d t+K(x(t)) d w$

where $x(t), u(t)$ and $y(t)$ take values in $\mathbb{R}^{n}, \mathbb{R}^{m}$ and, respectively, $\mathbb{R}^{p}$ and $w(t)$ is a Wiener process with values in $\mathbb{R}^{s}$ defined on a given probability space. We consider a family of controllers $C(k), k \in \mathbb{N}$,

$$
\begin{aligned}
u(t) & =\eta(F(k) \sigma(t)) \\
d \sigma(t) & =(Z(k) \sigma(t)+B u(t)) d t+G(k) d y(t)
\end{aligned}
$$

where $\eta: \mathbb{R}^{m} \rightarrow \mathbb{R}^{m}$ is a locally Lipschitz function, $F(k), G(k)$ and $Z(k)$ are matrices with suitable dimensions and the number $k$ parametrizes the desired region of attraction and target set for the closed-loop system (1)-(2). Thus, the parameters of controller (2) depend on the characteristics of the target set and the region of attraction. Denote by $z\left(t, t_{0}, z_{0}\right)=\operatorname{col}\left(x\left(t, t_{0}, x_{0}\right)\right.$, $\left.\sigma\left(t, t_{0}, \sigma_{0}\right)\right)$ the trajectory of the closed-loop system (1)(2) at time $t \geq t_{0}$ stemming from $z_{0}=\operatorname{col}\left(x_{0}, \sigma_{0}\right)$. With some abuse of notation, wherever there is no ambiguity, we will use $z(t)$ instead of $z\left(t, t_{0}, z_{0}\right)$. For any closed set $R \subset \mathbb{R}^{2 n}$ and open set $S \subset \mathbb{R}^{2 n}$ we denote by $\tau_{R \backslash S}$ the exit time of $z(t)$ from the set $R \backslash S$ and define $\tau_{R \backslash S}(t)=\min \left\{\tau_{R \backslash S}, t\right\}$. The following definition of stabilizability in probability for (1) has been given in [1].

Definition 1 Let $\alpha, \beta \in[0,1)$ and $R, T \subset \mathbb{R}^{2 n}$ be compact sets containing the origin. The system (1) is said to be $(R, T, \alpha, \beta)$-stabilizable in probability $($ or $(R, T, \alpha, \beta)$ $S P)$ if there exist a sequence of control laws $\{C(k)\}$, a sequence of compact sets $\left\{\Omega(k) \subset \mathbb{R}^{2 n}, k \in \mathbb{N}\right\}$ and open sets $\left\{S(k) \subset \mathbb{R}^{2 n}, k \in \mathbb{I N}\right\}$ such that

(i) there exists $k^{*} \in \mathbb{N}$ such that $\Omega(k) \supset R \supset T \supseteq S(k)$ for all $k \geq k^{*}$; 
(ii) for each $\delta>0$

$$
\begin{aligned}
& \liminf _{k \rightarrow \infty} \inf _{z_{0} \in \operatorname{clos}(S(k))} \mathbf{P}\left\{z(t) \in \operatorname{clos}\left(T_{\delta}\right) \forall t \geq t_{0}\right\} \\
& \geq 1-\beta
\end{aligned}
$$

(iii) for each $\delta>0$

$$
\begin{aligned}
& \liminf _{k \rightarrow \infty} \inf _{z_{0} \in R \backslash S(k)} \mathbf{P}\left\{z(t) \in \Omega(k) \forall t \geq t_{0},\right. \\
& \left.z\left(t+\tau_{\mathbb{R}^{2 n} \backslash S(k)}\right) \in \operatorname{clos}\left(T_{\delta}\right) \forall t \geq 0, \tau_{\mathbb{R}^{2 n} \backslash S(k)}<\infty\right\} \\
& \geq(1-\alpha)(1-\beta)
\end{aligned}
$$

The set $R$ gives the guaranteed region of attraction of the closed-loop system $\Sigma \circ C(k)$, while $T$ represents its target set. Property (ii) is a local property with respect to $T$ : for each $\delta$-neighbourhood $T_{\delta}$ of $T$, the probability that the trajectories $z(t)$ of the closed-loop system $\Sigma \circ C(k)$, starting from $\operatorname{clos}(S(k))$, stay forever in $\operatorname{clos}\left(T_{\delta}\right)$ is at least $1-\beta$ for sufficiently large $k$. Property (iii) is a property in the large with respect to $R$ : the trajectories of $\Sigma \circ C(k)$ starting inside $R$ remain inside $\Omega(k)$, eventually enter any given $\delta$-neighbourhood $T_{\delta}$ of the target set $T$ in finite time and remain thereinafter with probability at least $(1-\alpha)(1-\beta)$ for sufficiently large $k$.

The numbers $\alpha$ and $\beta$ are given risk margins: the first one quantifies the risk of leaving the compact set $\Omega(k)$ with initial condition in $\Omega$ rather than getting close to the target, while the second one gives a risk margin for remaining close to the target.

A technical condition for ensuring stabilization in probability with some target set $T$ and region of attraction $R$ for (1) is given by the following theorem ([1]): if there exists a $C^{1}$ proper and positive definite function $V$ such that along the trajectories of the closed-loop system resulting from (12), $\dot{V}$ is definite negative on $\Omega(k) \backslash S(k)$, where $\Omega(k)=\left\{z \in \mathbb{R}^{2 n}: V(z) \leq k\right\}$ and $\{S(k)\}$ is a sequence of open sets, containing the origin and contained in some level set of $V$, such that $S(k) \subseteq T$ for $k$ large, then any trajectory starting from $R \subseteq \Omega(k)$ stays forever in $\Omega(k)$, eventually enters any given neighbourhood of $T$ in finite time and remains thereinafter. For each (at least) $C^{2}$ function $V: \mathbb{R}^{2 n} \rightarrow \mathbb{R}$ be $L V(z)$ its infinitesimal generator along the trajectories of (1)-(2) ([5]).

Theorem 1 The system (1) is $(R, T, \alpha, \beta)-S P$ if there exist compact sets $R, T \subset \mathbb{R}^{2 n}$, containing the origin, a sequence of controllers $\{C(k), k \in \mathbb{N}\}$, a sequence of (at least) $C^{2}$, positive definite and proper $V: \mathbb{R}^{2 n} \rightarrow \mathbb{R}^{\geq}$, continuous positive definite $Q: \mathbb{R}^{2 n} \rightarrow \mathbb{R}^{\geq}$, open sets $\{S(k), k \in \mathbb{N}\}$ of $\mathbb{R}^{2 n}$ and $k^{*} \in \mathbb{N}$ such that for all $k \geq k^{*}$

$$
\begin{aligned}
& \text { (iv) } \Omega(k) \supset R \supset T \supseteq S(k), \text { where } \\
& \Omega(k)=\left\{z \in \mathbb{R}^{2 n}: V(z) \leq k\right\}
\end{aligned}
$$

(v) $L V(z) \leq-Q(z)$ for all $z \in \Omega(k) \backslash S(k)$;

$$
\begin{aligned}
& \text { (vi) } \sup _{z \in R \backslash S(k)} \frac{V(z)}{k} \leq \alpha \text { and } \frac{\sup _{z \in \partial S(k)} V(z)}{\inf _{z \in \mathbb{R}^{2 n} \backslash \operatorname{clos}\left(T_{\delta}\right)} V(z)} \leq \\
& \beta, \forall \delta>0
\end{aligned}
$$

In this case we also say that (1) is $(R, T, \alpha, \beta)-S P$ with Lyapunov function $V(z)$ and stability margin $Q(z)$.

The following fact, which will be used extensively in the next sections, follows from the proof of theorem 1 ([1]).

Lemma 1 Under assumptions of theorem 1 for any Markov time $t_{0}$ of the trajectories $z(t)$ of (1)-(2)

$$
\begin{aligned}
& \liminf _{k \rightarrow \infty} \mathbf{P}\left\{z\left(\tau_{R \backslash S(k)}(t)\right) \in \Omega(k), t \geq t_{0} ; z\left(t_{0}\right) \in R \backslash S(k)\right\} \\
& \geq(1-\alpha) \operatorname{Pr}\left\{z\left(t_{0}\right) \in R \backslash S(k)\right\}
\end{aligned}
$$

The conditions of theorem 1 can be met by first guaranteeing the existence of a state-feedback controller and then replacing the state with its estimate provided by an observer (see [1]). The following result stands as a "certainty equivalence principle" for nonlinear systems (1).

Theorem 2 Let $R, T \subset \mathbb{R}^{2 n}$ be compact sets containing the origin. Assume the existence of continuous $P_{s f}, Q_{s f}, P_{m}, Q_{m}: \mathbb{N} \rightarrow S P^{n}, R_{s f}: \mathbb{N} \rightarrow S P^{m}$, $R_{m}: \mathbb{N} \rightarrow S P^{p}, C^{1}$ function $\delta: \mathbb{R} \rightarrow(0,1], C^{0}$ locally Lipschitz $\eta: \mathbb{R}^{m} \rightarrow \mathbb{R}^{m}, \alpha, \beta \in[0,1)$, a sequence of open sets $\left\{S(k) \subset \mathbb{R}^{2 n}, k \in \mathbb{N}\right\}$ and $k^{*} \in \mathbb{N}$ such that for all $k \geq k^{*}$

- (state feedback) for all $x \in \Omega_{s f}(k):=\left\{v \in \mathbb{R}^{n}\right.$ : $\left.\|v\|_{P_{s f}(k)}^{2} \leq k\right\}$

$$
\begin{aligned}
& x^{T}\left[A^{T} P_{s f}(k)+P_{s f}(k) A\right] x-\left\|B^{T} P_{s f}(k) x\right\|_{R_{s f}^{-1}(k)}^{2} \\
& +\operatorname{Tr}\left\{H^{T}(x) P_{s f}(k) H(x)\right\} \leq-\|x\|_{Q_{s f}(k)}^{2}
\end{aligned}
$$

- (output injection ) $R_{m}(k) \geq K(x) K^{T}(x)$ for all $x \in \Omega_{s f}(k)$,

$$
\begin{aligned}
& P_{m}(k) A+A^{T} P_{m}(k)+P_{m}(k) H(x) H^{T}(x) P_{m}(k) \\
& -C^{T} R_{m}^{-1}(k) C+P_{s f}(k) B R_{s f}^{-1}(k) B^{T} P_{s f}(k) \\
& \leq-Q_{m}(k)
\end{aligned}
$$

and

$$
\lim _{s \rightarrow \infty} \psi(s)=\infty, \nabla_{s s}^{2} \psi(s) \leq \nabla_{s} \psi(s), \forall s \geq 0
$$

with $\psi(s)=\int_{0}^{s} \delta(\vartheta) d \vartheta$; 
- (coupling ) for all $x \in \Omega_{s f}(k)$ and $e \in \mathbb{R}^{n}$

$$
\begin{aligned}
& \|\eta(F(k)(x-e))-F(k) x\|_{R_{s f}(k)}^{2} \\
& +\sum_{j=1}^{r}\left[H_{j}^{T}(x) P_{m}(k) H_{j}(x)\right. \\
& \left.+K_{j}^{T}(x) R_{m}^{-1}(k) C P_{m}^{-1}(k) C^{T} R_{m}^{-1}(k) K_{j}(x)\right] \\
& -(1 / 2)\|x\|_{Q_{s f}(k)}^{2}-\delta\left(\|e\|_{P_{m}(k)}^{2}\right)\left[\|F(k) e\|_{R_{s f}(k)}^{2}\right. \\
& \left.\left.+(1 / 2)\|e\|_{Q_{m}(k)}^{2}\right)\right] \leq 0
\end{aligned}
$$

with $F(k)=-R_{s f}^{-1}(k) B^{T} P_{s f}(k)$ and $H_{j}(x)$ and $K_{j}(x)$ be the $j$-th column of $H(x)$ and $K(x)$, respectively;

- $\left(\right.$ risk margin) with $\Omega(k)=\left\{z \in \mathbb{R}^{2 n}: V(z) \leq k\right\}$

$$
S(k) \subseteq T \subset R \subset \Omega(k)
$$$$
\text { and for all } \delta>0 \text { with } V(z)=\|x\|_{P_{s f}(k)}^{2}+\psi(\| x-
$$$$
\left.\sigma \|_{P_{m}(k)}^{2}\right) \text { and }
$$

$$
\sup _{z \in R \backslash S(k)} \frac{V(z)}{k} \leq \alpha, \frac{\sup _{z \in \partial S(k)} V(z)}{\inf _{z \in \mathbb{R}^{2 n} \backslash \cos \left(T_{\delta}\right)} V(z)} \leq \beta
$$

Under the above assumptions, (1) is $(R, T, \alpha, \beta)-S P$ with Lyapunov function $V(z)$ and stability margin $Q(z)=$ $\|x\|_{Q_{s f}(k)}^{2}+\|e\|_{Q_{m}(k)}^{2} \delta\left(\|e\|_{P_{m}(k)}^{2}\right)$.

\section{Problem formulation and main results}

A switching system can be seen as a finite family of systems each one selected through a switching or time varying mechanism. In this paper we consider the following class of switching systems

$$
\begin{aligned}
d x(t) & =\left(A_{r(t)} x(t)+B_{r(t)} u(t)\right) d t+H_{r(t)}(x(t)) d w \\
d y(t) & =C_{r(t)} x(t) d t+K_{r(t)}(x(t)) d w
\end{aligned}
$$

where $x(t)$ and $u(t)$ take values in $\mathbb{R}^{n}$ and respectively $\mathbb{R}^{m}, y(t)$ takes values in $\mathbb{R}^{p}, w(t)$ is a Wiener process with values in $\mathbb{R}^{s}$ and $r(t)$ is a Markov chain taking values in $J=\{1, \ldots, N\}$. We denote by $t_{j_{l} j_{l+1}}$ the Markov (or switching) time at which $r(t)$ switches from $j_{l}$ to $j_{l+1}$ and define as dwell time the difference between two consecutive switching times. For simplicity, we denote $t_{j_{l} j_{l+1}}$ by $t_{j_{l+1}}$ and set $t_{0}:=0$. Moreover, the transition probabilities of the Markov chain are

$$
\begin{aligned}
& \operatorname{Pr}\left\{r(t+\Delta)=j_{l+1} \mid r(t)=j_{l}\right\}=\gamma_{j_{l} j_{l+1}} \Delta \text { if } j_{l} \neq j_{l+1}, \\
& \operatorname{Pr}\left\{r(t+\Delta)=j_{l+1} \mid r(t)=j_{l}\right\}=1+\gamma_{j_{l} j_{l}} \Delta \text { else }
\end{aligned}
$$

where $\gamma_{j_{l} j_{l}}=-\sum_{j_{l+1} \neq j_{l}} \gamma_{j_{l} j_{l+1}}$.

For each value of $r(t)=j$ we assume that there exist $\alpha_{j}, \beta_{j} \in[0,1)$ and two compact sets $R_{j}, T_{j} \subset \mathbb{R}^{2 n}$ such that (12) is $\left(R_{j}, T_{j}, \alpha_{j}, \beta_{j}\right)$-SP. In particular, we assume that the hypotheses of theorem 1 are met with a sequence of controllers $\left\{C_{j}(k), k \in \mathbb{N}\right\}$

$$
\begin{aligned}
u(t) & =\eta_{j}\left(F_{j}(k) \sigma(t)\right) \\
d \sigma(t) & =\left(Z_{j}(k) \sigma(t)+B_{j} u(t)\right) d t+G_{j}(k) d y(t)
\end{aligned}
$$

where $\eta_{j}: \mathbb{R}^{m} \rightarrow \mathbb{R}^{m}$ is a locally Lipschitz function. We assume that the Markov chain switches an infinite number of times, otherwise the stabilization problem of (12) is trivial because from the last switching of $r(t)$ from $j_{l}$ to $j_{l+1}$ the system (12) would be $\left(R_{j_{l+1}}, T_{j_{l+1}}, \alpha_{j_{l+1}}, \beta_{j_{l+1}}\right)^{-}$ SP. In this paper we study under which conditions a sequence of dwell-time switching controllers $\{C(k), k \in$ $\mathbb{N}\}$

$$
\begin{aligned}
u(t) & =\eta_{r(t)}\left(F_{r(t)}(k) \sigma(t)\right) \\
d \sigma(t) & =\left(Z_{r(t)}(k) \sigma(t)+B_{r(t)} u(t)\right) d t+G_{r(t)}(k) d y(t)(15)
\end{aligned}
$$

guarantees some stability properties of the closed-loop system. To this aim, we introduce a novel stability notion for which, given a decreasing family of sets $\left\{D_{i} \subset\right.$ $\left.\mathbb{R}^{2 n}: i \in \mathbb{N}\right\}$ approaching the target, the system (12)(15) is "stable under switching" if with an a priori given probability it happens that its trajectory $z(t)$ dwells a sufficiently large amount of time in $D_{i}$, before the next switching occurs, and approaches the set $D_{i+1}$. The fact that the trajectory goes through a decreasing sequence of sets $\left\{D_{i} \subset \mathbb{R}^{2 n}: i \in \mathbb{N}\right\}$ was already used as a condition for the stability analysis of deterministic hybrid systems in [11]. It is quite natural to require such "nesting property" of the sets $\left\{D_{i} \subset \mathbb{R}^{2 n}: i \in \mathbb{N}\right\}$, because if the trajectory of (12)-(15) is required to be stable and to approach the target in some reasonable sense then at each switching time this trajectory must belong to a decreasing sequence of sets which approach the target.

Definition 2 Given compact sets $R, T \subset \mathbb{R}^{2 n}$ and $\varrho \in$ $[0,1)$, the system (12) is said to be $(R, T, \varrho)$-stabilizable under switching (or $(R, T, \varrho)-S S$ ) if there exist a decreasing sequence of compact sets $D_{1}(k), \ldots, D_{l^{*}+1}(k) \in \mathbb{R}^{2 n}$ and $k^{*} \in \mathbb{N}$ for which $D_{l^{*}+1}(k) \subseteq T$ and $R \subseteq D_{1}(k)$ for all $k \geq k^{*}$ and a sequence of feedback control laws $\{C(k), k \in \mathbb{N}\}$ of the form (15) such that for all $k \geq k^{*}$

$\operatorname{Pr}\{\Xi(k)\} \geq(1-\varrho) \operatorname{Pr}\{z(0) \in R \backslash T\}$

where $\Xi(k)$ is the following event

$$
\begin{aligned}
& \Xi(k)=\left\{z\left(\tau_{\mathbb{R}^{2 n} \backslash T}(t)\right) \in D_{l}(k), t \geq \tau_{\mathbb{R}^{2 n} \backslash T}\left(t_{j_{l}}\right),\right. \\
& \left.z\left(\tau_{\mathbb{R}^{2 n} \backslash T}\left(t_{j_{l+1}}\right)\right) \in D_{l+1}(k), z(0) \in R \backslash T, l=1, \ldots, l^{*}\right\}
\end{aligned}
$$

In this case we also say that (12) is $(R, T, \varrho)-S S$ with switching controllers $\{C(k), k \in \mathbb{N}\}$.

Note that the trajectories are stopped when $z(t) \in T$ since we consider of interest only the event for which the trajectory $z(t)$ may dwell outside the target. 


\section{$5 \quad$ Switching dwell-time controllers}

A first result to be established is, given a $C^{2}$ Lyapunov function $V_{i}: \mathbb{R}^{2 n} \rightarrow \mathbb{R}^{\geq}$for the "frozen" system (12), what is the expression of $L V_{i}$. It turns out that, if $V_{i}$ a Lyapunov function for (1) when $r(t)=i$, it has the same expression as if there were no switching plus a "weighted average" term of the functions $V_{j}, j \in J$, weighted by the transition probabilities of the Markov chain.

\section{Lemma 2 Let}

$d z=a_{r(t)}(z) d t+b_{r(t)}(z) d w$

where $z(t)$ takes values in $\mathbb{R}^{2 n}$, w(t) is a Wiener process with values in $\mathbb{R}^{s}, r(t)$ is a Markov chain taking values in $J$ and $a_{i}: \mathbb{R}^{2 n} \rightarrow \mathbb{R}^{2 n}$ and $b_{i}: \mathbb{R}^{2 n} \rightarrow \mathbb{R}^{2 n \times s}$ locally Lipschitz functions. If $V_{i}(z)$ is for each $i \in J$ a smooth function, then

$$
\begin{aligned}
& L V_{i}(z(t))=\lim _{\Delta \rightarrow 0^{+}} \frac{1}{\Delta}\left[\mathbf{E}\left\{V_{r(t+\Delta)}(z(t+\Delta)) \mid z(t) ; r(t)=i\right\}\right. \\
& \left.-V_{i}(z(t))\right]=\nabla_{z} V_{i}(z(t), i) a_{i}(z(t))+\sum_{j=1}^{N} \gamma_{i j} V_{j}(z(t)) \\
& +\frac{1}{2} \operatorname{Tr}\left\{b_{i}^{T}(z(t)) \nabla_{z z}^{2} V_{i}(z(t)) b_{i}(z(t))\right\}
\end{aligned}
$$

Proof. We follow the lines of [10]. We have by definition of $\gamma_{i j}$

$$
\begin{aligned}
& \mathbf{E}\left\{V_{r(t+\Delta)}(z(t+\Delta)) \mid z(t) ; r(t)=i\right\} \\
& =\sum_{j=1}^{N} \operatorname{Pr}\{r(t+\Delta)=j \mid z(t) ; r(t)=i\} V_{j}(z(t)) \\
& +\mathbf{E}\left\{V_{r(t+\Delta)}(z(t+\Delta)) \mid z(t) ; r(t)=i\right\} \\
& -\mathbf{E}\left\{V_{r(t+\Delta)}(z(t)) \mid z(t) ; r(t)=i\right\} \\
& =\sum_{j=1}^{N} \Delta \gamma_{i j} V_{j}(z(t))+V_{i}(z(t)) \\
& +\mathbf{E}\left\{V_{r(t+\Delta)}(z(t+\Delta)) \mid z(t) ; r(t)=i\right\} \\
& -\mathbf{E}\left\{V_{r(t+\Delta)}(z(t)) \mid z(t) ; r(t)=i\right\}
\end{aligned}
$$

Moreover, by (4.88) of [6] with $f(s, z):=V_{i}(z)$ and $s=0$, for all $\xi \geq 0$

$$
\begin{aligned}
& \mathbf{E}\left\{V_{r(t+\Delta)}(z(\xi)) \mid z(t) ; r(t)=i\right\} \\
& =\mathbf{E}\left\{\int _ { 0 } ^ { \xi } \left[\nabla_{z} V_{i}(z(\theta)) a_{i}(z(\theta))\right.\right. \\
& \left.\left.+\frac{1}{2} \operatorname{Tr}\left\{b_{i}^{T}(z(\theta)) \nabla_{z z}^{2} V_{i}(z(\theta)) b_{i}(z(\theta))\right\}\right] d \theta\right\}
\end{aligned}
$$

From (20) with $\xi=t+\Delta$ and, respectively, $\xi=t$, dividing by $\Delta$ and passing to the limit for $\Delta \rightarrow 0$ we obtain (18) from (19).

Using the previous lemma we can prove one of the main results of this paper. If the level sets of the Lyapunov function $V_{i}$ of each "frozen" $i$-th system (12)-(15) satisfy a suitable "nesting property" and the expectation of the dwell time is sufficiently large, conditionally to the trajectories of (12)-(15) ensuing from the level set of $V_{i}$ and dwelling therein till the next switching, then the system (12)-(15) is stable under switching in the sense of definition 2 with a guaranteed probability depending on the risk margins of each frozen system (12)-(15).

Theorem 3 For $j \in J$ let $R_{j}, T_{j} \subset \mathbb{R}^{2 n}$ be compact sets, $T=\cup_{j=1}^{N} T_{j}, R=\cup_{j=1}^{N} R_{j}$ and $\alpha_{j}, \beta_{j} \in[0,1)$ such that (12), with $r(t)=j$, is $\left(R_{j}, T_{j}, \alpha_{j}, \beta_{j}\right)$ $S P$ with Lyapunov function $V_{j}(z)$, stability margin $Q_{j}(z):=\nu_{j}\left(V_{j}(z)\right)+\sum_{l=1}^{N} \gamma_{j l} V_{l}(z)$ for some $\nu_{j} \in K$, smooth over $(0, \infty)$, and controllers $\left\{C_{j}(k), k \in \mathbb{N}\right\}$ and let $S(k)=\cup_{j=1}^{N} S_{j}(k)$, where the sets $S_{j}(k)$ are given by the definition of $\left(R_{j}, T_{j}, \alpha_{j}, \beta_{j}\right)-S P$. Assume the existence of $k^{*} \in \mathbb{N}, l^{*} \in \mathbb{N}$ and continuous $\varphi_{j_{l} j_{l+1}}, a_{j_{l}}: \mathbb{N} \rightarrow \mathbb{R}^{+}$such that for all $k \geq k^{*}$ and $l \in\left[1, l^{*}\right]$

- (level nesting) with $\Omega_{j_{l}}(k):=\left\{z \in \mathbb{R}^{2 n}: V_{j_{l}}(z) \leq\right.$ $k\}$ and for all $j_{l}, j_{l+1} \in J$

$$
\Omega_{j_{l+1}}(k) \cap \Omega_{j_{l}}(k) \supset \Omega_{j_{l}}\left(\varphi_{j_{l} j_{l+1}}(k)\right)
$$

and there exists $l^{*} \in \mathbb{N}$ such that for all $j_{1}, \ldots, j_{l^{*}} \in J$

$$
S(k) \subset \Omega_{j_{1}}\left(\Phi_{j_{l^{*}}}(k)\right) \subset T
$$

where $\Phi_{j_{l}}(k)=\varphi_{j_{l} j_{l+1}} \circ \cdots \varphi_{j_{2} j_{3}} \circ \varphi_{j_{1} j_{2}}(k), \Phi_{j_{0}}(k)=k$ and $\varphi_{j j}(k)=k$;

- (minimum expected dwell time) there exists $\epsilon_{j_{l}} \in$ $[0,1)$ such that for all $j_{l-1}, j_{l} \in J$

$$
\begin{aligned}
& \mathbf{E}\left\{\tau_{\mathbb{R}^{2 n} \backslash S(k)}\left(t_{j_{l}}\right)-\tau_{\mathbb{R}^{2 n} \backslash S(k)}\left(t_{j_{l-1}}\right) \mid \Lambda_{j_{l-1}}(k)\right\} \\
& \geq I_{j_{l}}\left(\Phi_{j_{l-1}}(k)\right)-\epsilon_{j_{l}} I_{j_{l}}\left(\Phi_{j_{l}}(k)\right)
\end{aligned}
$$

where $I_{j_{l}}(s)=\int_{a_{j_{l}}(k)}^{s}\left(1 / \nu_{j_{l}}(r)\right) d r$ for $s>a_{j_{l}}(k)$ and $\Omega_{j_{l}}\left(a_{j_{l}}(k)\right) \subset S_{j_{l}}(k)$, and the events $\Lambda_{j_{l}}$ are defined as follows

$$
\begin{aligned}
\Lambda_{j_{l}}(k)= & \left\{z\left(\tau_{\mathbb{R}^{2 n} \backslash S(k)}(t)\right) \in \Omega_{j_{1}}\left(\Phi_{j_{l}}(k)\right)\right. \\
& \left.t \geq \tau_{\mathbb{R}^{2 n} \backslash S(k)}\left(t_{j_{l}}\right)\right\} \\
\Lambda_{j_{0}}(k)= & \left\{z\left(\tau_{\mathbb{R}^{2 n} \backslash S(k)}(t)\right) \in \Omega_{j_{1}}(k)\right. \\
& \left.t \geq 0, z(0) \in \Omega_{j_{1}}(k) / S(k)\right\}
\end{aligned}
$$

Under the above assumptions, (12) is (R,T,1 $\left.\Pi_{l=1}^{l^{*}}\left(1-\epsilon_{j_{l}}\right)\left(1-\alpha_{j_{l}}\right)\right)-S S$ with switching controllers $\left\{C_{r(t)}(k), k \in \mathbb{N}\right\}$. 
Remark. Theorem 3 states that if the expectation of the dwell-time is "sufficiently large" and the sets $\left\{\Omega_{j_{l}}(k)\right\}$ satisfy a "nesting property" then the switching system is stable under switching with guaranteed probability. This condition generalizes to a stochastic framework the results for deterministic switching systems, for which the dwell time must be long enough to let the system trajectory to go through a decreasing sequence of sets ([7], [8], [9], [11])). The expectation (23) has to be computed conditionally to the event $\Lambda_{j_{l}}(k)$. Although this may be difficult to do, it comes directly from the definition of stability under switching itself, for which only the event $\Lambda_{j_{l}}(k)$ is significant during each dwell time $t_{j_{l+1}}-t_{j_{l}}$ and it happens with a given probability.

Remark. From (23) it is clear that $\epsilon_{j}$ can be interpreted as the risk that the trajectory does not enter $\Omega_{j_{1}}\left(\Phi_{j_{l}}(k)\right)$ starting from $\Omega_{j_{1}}\left(\Phi_{j_{l-1}}(k)\right)$ : the longer is the expected dwell time $\mathbf{E}\left\{\tau\left(t_{j_{l}}\right)-\tau\left(t_{j_{l-1}}\right) \mid \Lambda_{j_{l-1}}(k)\right\}$ to be awaited the smaller is the risk $\varepsilon_{j}$.

Proof. Throughout the proof we omit the argument $k$, denote $\tau_{\mathbb{R}^{n} \backslash S}(t)$ simply by $\tau(t)$ and let $j \in J$. Assume that $k^{*} \in \mathbb{N}$ is chosen in such a way that

$R_{j} \subset \Omega_{j}, S_{j} \subset T_{j}$

for all $k \geq k^{*}$. Let $j \in J$. Since $(12)$ is $\left(R_{j}, T_{j}, \alpha_{j}, \beta_{j}\right)-$ SP with Lyapunov function $V_{j}(z)$, stability margin $Q_{j}(z):=\nu_{j}\left(V_{j}(z)\right)+\sum_{l=1}^{N} \gamma_{j l} V_{j}(z)$ and controllers $\left\{C_{j}\right\}$, by lemma 2 , then along the trajectories of (12) with controllers $\left\{C_{j}\right\}$

$$
L V_{j} \leq-\nu_{j}\left(V_{j}(z)\right), \forall z \in \Omega_{j} \backslash S_{j}
$$

where $L V_{j}$ is defined as in (18) for (12) with controller $\left\{C_{r(t)}\right\}$.

Moreover, let $W_{j}(z, t)=I_{j}\left(V_{j}(z)\right)+t$. By the Ito rule and $(25)$, since $\nabla_{s s}^{2} I_{j}(s) \leq 0$ for all $s \in\left(a_{j_{l}}, \infty\right)$ then

$L W_{j}(z) \leq\left.\frac{\partial I_{j}}{\partial s}\right|_{s=V_{j}(z)} L V_{j}(z)+1 \leq 0$

for all $z \in \Omega_{j} \backslash S_{j}$. Along the trajectories of (12) with controller $\left\{C_{j_{1}}\right\}$ and from (26) with $j=j_{1}$

$L W_{j_{1}} \leq 0, \forall z \in \Omega_{j_{1}} \backslash S_{j_{1}}$

Define the following event

$\Xi_{j_{0}}=\Lambda_{j_{0}} \cap\left\{z\left(\tau\left(t_{j_{1}}\right)\right) \in \Omega_{j_{1}}\left(\varphi_{j_{1} j_{2}}\right)\right\}$

Note that by lemma 1

$\operatorname{Pr}\left\{\Lambda_{j_{0}}\right\} \geq\left(1-\alpha_{j_{1}}\right) \operatorname{Pr}\left\{z(0) \in \Omega_{j_{1}} \backslash S\right\}$
By Dynkin's formula and (27)

$$
\mathbf{E}\left\{W_{j_{1}}(z(\tau(t))) \mid \Lambda_{j_{0}}\right\} \leq \mathbf{E}\left\{W_{j_{1}}(x(0)) \mid \Lambda_{j_{0}}\right\}, \forall t \geq 0
$$

By definition of $W_{j_{1}}$ and (30)

$$
\begin{aligned}
& \mathbf{E}\left\{I_{j_{1}}\left(V_{j_{1}}(z(\tau(t)))\right) \mid \Lambda_{j_{0}}\right\} \leq \mathbf{E}\left\{I_{j_{1}}\left(V_{j_{1}}(z(0))\right) \mid \Lambda_{j_{0}}\right\} \\
& -\mathbf{E}\left\{\tau(t) \mid \Lambda_{j_{0}}\right\}, \forall t \geq 0
\end{aligned}
$$

Note that $I_{j_{1}}(s)$ is strictly increasing for all $s>a_{j}$ and then $\mathbf{E}\left\{I_{j_{1}}\left(V_{j_{1}}(z(0)) \mid \Lambda_{j_{0}}\right\} \leq I_{j_{1}}(k)\right.$ since $\operatorname{Pr}\{x(0) \in$ $\left.\Omega_{j_{1}} \backslash S \mid A_{j_{0}}\right\}=1$. By Cebyšev and (31)

$$
\begin{aligned}
& \operatorname{Pr}\left\{z\left(\tau\left(t_{j_{1}}\right)\right) \notin \Omega_{j_{1}}\left(\varphi_{j_{1} j_{2}}\right) \mid \Lambda_{j_{0}}\right\} \\
& =\operatorname{Pr}\left\{I_{j_{1}}\left(V_{j_{1}}(z(\tau))>I_{j_{1}}\left(\varphi_{j_{1} j_{2}}\right) \mid \Lambda_{j_{0}}\right\}\right. \\
& \leq\left[1 / I_{j_{1}}\left(\varphi_{j_{1} j_{2}}\right)\right]\left[I_{j_{1}}(k)-\mathbf{E}\left\{\tau\left(t_{j_{1}}\right) \mid \Lambda_{j_{0}}\right\}\right]
\end{aligned}
$$

It follows from (32) and (29) that

$$
\begin{aligned}
& \mathbf{E}\left\{\tau\left(t_{j_{1}}\right) \mid \Lambda_{j_{0}}\right\} \geq I_{j_{1}}(k)-\epsilon_{j_{1}} I_{j_{1}}\left(\varphi_{j_{1} j_{2}}\right) \\
& \Rightarrow \operatorname{Pr}\left\{\Xi_{j_{0}}\right\} \geq\left(1-\epsilon_{j_{1}}\right)\left(1-\alpha_{j_{1}}\right) \operatorname{Pr}\left\{z(0) \in \Omega_{j_{1}}\right\}
\end{aligned}
$$

Let $h \in\left[1, l^{*}-1\right]$. Define the following event

$\Xi_{j_{l}}=\Lambda_{j_{l}} \cap\left\{z\left(\tau\left(t_{j_{l+1}}\right) \in \Omega_{j_{l+1}}\left(\Phi_{j_{l+1}}\right)\right\}\right.$

for $l=1, \ldots, l^{*}$. We prove by induction that

$$
\begin{aligned}
& \mathbf{E}\left\{\tau\left(t_{j_{l}}\right)-\tau\left(t_{j_{l-1}}\right) \mid \Lambda_{j_{l-1}}\right\} \\
& \left.\geq I_{j_{l}}\left(\Phi_{j_{l-1}}\right)-\epsilon_{j_{l}} I_{j_{l}}\left(\Phi_{j_{l}}\right)\right], l \in[1, h+1] \\
& \Rightarrow \operatorname{Pr}\left\{\Xi_{j_{l-1}}\right\} \geq \Pi_{r=1}^{l}\left(1-\epsilon_{j_{r}}\right)\left(1-\alpha_{j_{r}}\right) . \\
& \cdot \operatorname{Pr}\left\{z(0) \in \Omega_{j_{1}} \backslash S\right\}
\end{aligned}
$$

Assume that (35) holds true for all integers $l \in[1, h]$. Note that by lemma 1

$\operatorname{Pr}\left\{\Lambda_{j_{h}}\right\} \geq\left(1-\alpha_{j_{h+1}}\right) \operatorname{Pr}\left\{z\left(\tau\left(t_{j_{h}}\right) \in \Omega_{j_{1}}\left(\Phi_{j_{h}}\right)\right\}\right.$

Taking into account that

$\operatorname{Pr}\left\{\Xi_{j_{h-1}}\right\} \leq \operatorname{Pr}\left\{z\left(\tau\left(t_{j_{h}}\right) \in \Omega_{j_{1}}\left(\Phi_{j_{h}}\right)\right\}\right.$

and that by the level nesting assumption $(21) \Omega_{j_{1}}\left(\Phi_{j_{h}}\right)$ $\subset \Omega_{j_{h+1}}$, reasoning as above in (33) we obtain for each $\epsilon_{j_{h+1}} \in[0,1)$

$$
\begin{aligned}
& \left.\mathbf{E}\left\{\tau\left(t_{j_{h+1}}\right)-\tau\left(t_{j_{h}}\right) \mid \Lambda_{j_{h}}\right\} \geq I_{j_{h+1}}\left(\Phi_{j_{h}}\right)-\epsilon_{j_{h+1}} I_{j_{h}}\left(\Phi_{j_{h}}\right)\right] \\
& \Rightarrow \operatorname{Pr}\left\{\Xi_{j_{h}}\right\} \geq\left(1-\epsilon_{j_{h+1}}\right)\left(1-\alpha_{j_{h+1}}\right) \operatorname{Pr}\left\{\Xi_{j_{h-1}}\right\}
\end{aligned}
$$

From (37) and the induction step we get (35).

Using (22) and (24) and defining $D_{1}=\cup_{j=1}^{N} \Omega_{j}, D_{l}=$ $\left.\Omega_{j_{1}}\left(\Phi_{j_{l-1}}\right)\right), l=2, \ldots, l^{*}+1$, this proves that (12) with 
controller $\left\{C_{r(t)}, k \in \mathbb{N}\right\}$ is $\left(R, T, 1-\Pi_{l=1}^{l^{*}}\left(1-\epsilon_{l}\right)(1-\right.$ $\left.\left.\alpha_{l}\right)\right)$-SS.

As an application of theorem 3we want to study the case of switching linear systems. For $j \in J$ let $R_{j}, T_{j} \subset \mathbb{R}^{2 n}$ be compact sets and $\alpha_{j}, \beta_{j} \in[0,1)$ and assume that

$d x(t)=\left(A_{r(t)} x(t)+B_{r(t)} u(t)\right) d t+H_{r(t)} x(t) d w$

$d y(t)=C_{r(t)} x(t) d t+K_{r(t)} x(t) d w$

with $r(t)=j$, is $\left(R_{j}, T_{j}, \alpha_{j}, \beta_{j}\right)$-SP with quadratic Lyapunov functions $V_{j}(z)=(1 / 2)\|z\|_{P_{j}}^{2}$ where $P_{j}$ is symmetric and positive definite, quadratic stability margins $Q_{j}(z):=\nu_{j} V_{j}(z)+\sum_{l=1}^{N} \gamma_{j l} V_{l}(z)$ for some $\nu_{j}>0$, and linear controller $C_{j}$ (independent of $k$ ). This assumption is quite natural for the class of systems (38) according to the results of [1]. It is also reasonable to assume that $S_{j}(k)$ are independent of $k$ and equal to int $\left(\Omega_{j}\left(c_{j}\right)\right)$ for some $c_{j}>0$. Set $k \geq \max _{j} c_{j}$. By definition, $\Omega_{j_{l}}(k):=\left\{z \in \mathbb{R}^{2 n}:(1 / 2)\|z\|_{P_{j_{l}}}^{2} \leq k\right\}$ and

$$
\begin{aligned}
& \Omega_{j_{l+1}}(k) \cap \Omega_{j_{l}}(k) \supset\left\{z \in \mathbb{R}^{2 n}:\|z\|^{2}\right. \\
& \leq k \min \left\{\lambda_{\max }^{-1}\left(P_{j_{l}}\right), \lambda_{\max }^{-1}\left(P_{j_{l+1}}\right)\right\}
\end{aligned}
$$

If the functions $\varphi_{j_{l} j_{l+1}}: \mathbb{N} \rightarrow \mathbb{R}^{+}$are defined so that

$\varphi_{j_{l} j_{l+1}}(k)=k \gamma_{l} \min \left\{\lambda_{\max }^{-1}\left(P_{j_{l+1}}\right), \lambda_{\max }^{-1}\left(P_{j_{l}}\right)\right\}$

with $\gamma_{l} \in(0,1)$, then $\Omega_{j_{l+1}}(k) \cap \Omega_{j_{l}}(k) \supset \Omega_{j_{l}}\left(\varphi_{j_{l} j_{l+1}}(k)\right)$, i.e. $(21)$. For $j_{l} \in J$ define

$$
\begin{aligned}
& \Phi_{j_{l}}(k):=\varphi_{j_{l} j_{l+1}} \circ \cdots \varphi_{j_{2} j_{3}} \circ \varphi_{j_{1} j_{2}}(k) \\
& =k \Pi_{i=1}^{l} \gamma_{i} \min \left\{\lambda_{\max }^{-1}\left(P_{j_{i+1}}\right), \lambda_{\text {max }}^{-1}\left(P_{j_{i}}\right)\right\}
\end{aligned}
$$

and $\Phi_{0}(k)=k$. It is always possible to choose $l^{*} \in \mathbb{N}, k^{*} \in \mathbb{N}$ and $\gamma_{1}, \ldots, \gamma_{l^{*}} \in(0,1)$ such that $\Omega_{j_{1}}\left(\Phi_{j^{*}}(k)\right) \subset T:=\cup_{j=1}^{N} T_{j}, S_{j}(k) \subset \Omega_{j}(k)$ and $S(k):=\cup_{j=1}^{N} S_{j}(k) \subset \Omega_{j_{1}}\left(\Phi_{j_{l^{*}}}(k)\right)$ for all $j_{1}, \ldots, j_{l^{*}} \in J$ and for all $k \geq k^{*}$, which proves (22). Next, define $I_{j}(s):=\int_{c_{j} / 2}^{s}\left(1 / \nu_{j} s\right) d s=\left(1 / \nu_{j}\right) \log \left(2 s / c_{j}\right)$, with $s>c_{j} / 2$. For each $j_{l} \in J$ and $\epsilon_{j_{l}} \in[0,1)$, we obtain by direct calculations

$$
\begin{aligned}
& I_{j_{l}}\left(\Phi_{j_{l-1}}(k)\right)-\epsilon_{j_{l}} I_{j_{l}}\left(\Phi_{j_{l}}(k)\right) \\
& =\left(1 / \nu_{j_{l}}\right)\left\{\log \left(2 \Phi_{j_{l-1}}(k) / c_{j_{l-1}}\right)-\epsilon_{j_{l}} \log \left(2 \Phi_{j_{l}}(k) / c_{j_{l}}\right)\right\}
\end{aligned}
$$

Thus, by applying theorem 3, we conclude that (38) is $\left(R, T, 1-\Pi_{l=1}^{l^{*}}\left(1-\epsilon_{j_{l}}\right)\left(1-\alpha_{j_{l}}\right)\right)$-SS with switching controllers $C_{r(t)}$ (independent of $k$ ) if

$$
\begin{aligned}
& \mathbf{E}\left\{\tau\left(t_{j_{l}}\right)-\tau\left(t_{j_{l-1}}\right) \mid \Lambda_{j_{l-1}}(k)\right\} \\
& \geq\left(1 / \nu_{j_{l}}\right)\left\{\log \left(2 \Phi_{j_{l-1}}(k) / c_{j_{l-1}}\right)-\epsilon_{j_{l}} \log \left(2 \Phi_{j_{l}}(k) / c_{j_{l}}\right)\right\}
\end{aligned}
$$

\section{Applications}

In this section we use the methodology introduced in the previous sections to design a switching controller for the following class of systems

$$
\begin{aligned}
d x_{j} & =x_{j+1} d t, j=1, \ldots, n-1, \\
d x_{n} & =u d t+h_{r(t)}(x) d w \\
y & =x_{1}
\end{aligned}
$$

where $x=\operatorname{col}\left(x_{1}, \ldots, x_{n}\right), x_{i} \in \mathbb{R}, u \in \mathbb{R}$ is the control, $y \in \mathbb{R}$ is the measurement, $w \in \mathbb{R}$ is a Wiener process and $r \in J:=1, \ldots, N$ a Markov chain. Moreover, we assume that $h_{r}(x)$ is for each $r$ a locally Lipschitz function, vanishing at the origin. In [1] a measurement feedback controller (14) has been designed using control saturations and a logarithmic Lyapunov function for the state estimation error system. Control saturations avoid the "peaking" of some state variables, while a logarithmic Lyapunov function for the state estimation error system allows to enlarge the region of attraction of the closedloop system. On the other hand, following the results of [3], one can choose other Lyapunov functions than logarithmic ones to enlarge the region of attraction. This determines through a suitable "coupling condition" the choice of the function $\eta_{j}(\cdot)$ in (14). Our main result is to prove that in the presence of a Markov chain the choice of the Lyapunov function for the state estimation error system cannot be logarithmic, due to the "weighted average" in (18) of the Lyapunov functions of each frozen system, weighted by the transition probabilities of the Markov chain. Thus, it is necessary to design the Lyapunov function of the state estimation error system in order to compensate for the effect of the weighted average term in (14) and at the same time to enlarge the region of attraction of the closed-loop system: as a result we obtain a Lyapunov function $V_{m}(e)=\left(1+\|e\|^{2}\right)^{1 / r}-1$ where $r \geq 4(n-1)+1$ and, according to the "coupling condition", a function $\eta_{j}(s)$ which is linear over the region of attraction and goes as $s^{1 / r}$ outside.

To find a switching measurement feedback controller for (42) we adopt a certainty equivalence principle: first, we design a switching state feedback controller, then we replace the state in this controller with a suitable estimate given by an observer.

\subsection{Backstepping design}

The following result can be proved as in [2], theorem 5.1. Let $\pi=\operatorname{col}\left(x_{1}, \ldots, x_{n-1}\right), x=\operatorname{col}\left(\pi, x_{n}\right)$ and $A_{0}$ and $B_{0}$ be matrices such that (42) rewrites as

$$
\begin{gathered}
d \pi=\left(A_{0} \pi+B_{0} u\right) d t \\
d x_{n}=u d t+h_{r(t)}(x) d w
\end{gathered}
$$

Theorem 4 Let $j, i \in J$ and $T_{j} \subset R_{j} \subset \mathbb{R}^{n}$ be compact sets, $\left\{S_{j}(k) \subset \mathbb{R}^{n}, k \in \mathbb{N}\right\}$ a sequence of open sets such 
that $S_{j}(k) \subset T_{j}$ for $k$ large, with $S(k)=\cup_{j=1}^{N} S_{j}(k)$, and $\alpha_{j} \in[0,1)$. Therefore, there exist $l^{*}, k^{*} \in \mathbb{I}$, continuous $\Delta, a_{j}: \mathbb{N} \rightarrow \mathbb{R}^{+}$, positive definite $V_{j}: \mathbb{R}^{n} \rightarrow \mathbb{R}^{\geq}$, $j \in J$, and a family of linear state feedback controllers $\left\{C_{j}(k), k \in \mathbb{N}\right\}, j \in J$, such that, if for all $j_{l}, j_{l+1} \in J$ and $k \geq k^{*}$

$\mathbf{E}\left\{\tau_{\mathbb{R}^{n} \backslash S(k)}\left(t_{j_{l+1}}\right)-\tau_{\mathbb{R}^{n} \backslash S(k)}\left(t_{j_{l}}\right) \mid \Lambda_{j_{l}}(k)\right\} \geq \Delta(k)$

with $\Lambda_{j_{l}}(k)$ and $\tau_{\mathbb{R}^{n} \backslash S(k)}(t)$ defined as in theorem 3 with $z=x$, then for all $k \geq k^{*}$ (42) is $\left(\cup_{l=1}^{N} R_{l}, \cup_{l=1}^{N} T_{l}, 1-\right.$ $\left.\Pi_{i=1}^{l^{*}}\left(1-\alpha_{i}\right)\right)-S S$ with state feedback switching controllers $\{C(k), k \in \mathbb{N}\}=\left\{C_{r(t)}(k), k \in \mathbb{N}\right\}$.

Proof. Let the linear controllers $C_{j}(k): u=-\lambda_{j}^{2}(k)$ $\widetilde{R}_{s f j}^{-1}(k) \widetilde{P}_{s f j}(k)\left(x_{n}+B_{0}^{T} P \pi\right), j=1, \ldots, N$, with continuous $\widetilde{P}_{s f j}, \widetilde{R}_{s f j}, \lambda_{j}: \mathbb{N} \rightarrow \mathbb{R}^{+}$and $P \in S P^{(n-1) \times(n-1)}$ designed as in section V.A of [2] for each frozen system (43) in such a way that $\lim _{k \rightarrow \infty} \widetilde{P}_{s f j}(k) / k=0$, $\lim _{k \rightarrow \infty} \lambda_{j}(k)=0$ and

$A_{0}^{T} P+P A_{0}-P B_{0} B_{0}^{T} P+I=0$

Moreover, according to section V.A of [2] we have a quadratic Lyapunov function $V_{s f j}(z)=(1 / 2)\|\pi\|_{P}^{2}+$ $\lambda_{j}^{2}(k) \widetilde{P}_{s f j}(k)\left(x_{n}+B_{0}^{T} P \pi\right)^{2}$ and a quadratic stability $\operatorname{margin} Q_{s f j}(z)=(1 / 2)\left[\|\pi\|^{2}+\left(x_{n}+B_{0}^{T} P \pi\right)^{2} \widetilde{Q}_{s f j}(k)\right]$ for some continuous $\widetilde{Q}_{s f j}: \mathbb{N} \rightarrow \mathbb{R}^{+}$such that $\widetilde{Q}_{s f j}(k) \geq 2 / \lambda_{j}(k)$. We claim that it is possible to select $\widetilde{Q}_{s f j}: \mathbb{N} \rightarrow \mathbb{R}^{+}$and $k^{*} \in \mathbb{I}$ in such a way to have $Q_{s f j}(z)=\nu_{s f j} V_{s f j}(z)+\sum_{i=1}^{N} \gamma_{j i} V_{s f i}(z)$ for some $\nu_{s f j}>0$ and for all $k \geq k^{*}$. Indeed, using the fact that $\sum_{i} \gamma_{j i}=0$ for each $j$ and that $\lim _{k \rightarrow \infty} \lambda_{j}(k)=0$ we get

$$
\begin{aligned}
& Q_{s f j}(z)=(1 / 2)\left[\|\pi\|^{2}+\left(x_{n}+B_{0}^{T} P \pi\right)^{2} \widetilde{Q}_{s f j}\right] \\
& \geq\left(1 / \lambda_{\max }(P)\right) V_{s f j}(z)+\sum_{i=1}^{N} \gamma_{j i} V_{s f i}(z)
\end{aligned}
$$

Thus, let $\nu_{s f j}=1 / \lambda_{\max }(P)$ and $\widetilde{Q}_{s f j}(k) \geq \max \left\{2 / \lambda_{j}(k)\right.$, $\left.\left(\nu_{s f j}+\gamma_{j j}\right) \lambda_{j}^{2}(k) \widetilde{P}_{s f j}(k)+\sum_{i \neq j} \gamma_{j i} \lambda_{i}^{2}(k) \widetilde{P}_{s f i}(k)\right\}$, which proves our claim. Moreover, we have $\Omega_{s f j}(k)=\{x$ : $\left.(1 / 2)\|x\|_{P_{s f j}(k)}^{2} \leq k\right\}$ where

$$
\begin{aligned}
& P_{s f j}(k)= \\
& \left(\begin{array}{cc}
P+\lambda_{j}^{2}(k) \widetilde{P}_{s f j}(k) P B_{0} B_{0}^{T} P & -\lambda_{j}^{2}(k) \widetilde{P}_{s f j}(k) P B_{0} \\
-\lambda_{j}^{2}(k) \widetilde{P}_{s f j}(k) B_{0}^{T} P & \lambda_{j}^{2}(k) \widetilde{P}_{s f j}(k)
\end{array}\right)
\end{aligned}
$$

Thus, to prove the assumptions of theorem 3 we can proceed exactly as at the end of section 5 for linear systems with $\epsilon_{j}=0, j \in J$.

\section{2 $H_{\infty}$ filtering}

In this section, we design an observer for estimating the state and replace the state in the controller $\{C(k), k \in$ $\mathbb{N}\}=\left\{C_{r(t)}(k), \quad k \in \mathbb{N}\right\}$ of theorem 4 to obtain a measurement feedback controller. Rewrite (43) as

$d x=(A x+B u) d t+h_{r(t)}(x) d w$

$d y=C x d t$

where

$$
A=\left(\begin{array}{cccccc}
0 & 1 & 0 & \cdots & 0 & 0 \\
0 & 0 & 1 & \cdots & 0 & 0 \\
\vdots & \vdots & \vdots & \cdots & \vdots & \vdots \\
0 & 0 & 0 & \cdots & 0 & 1 \\
0 & 0 & 0 & \cdots & 0 & 0
\end{array}\right), B=\left(\begin{array}{c}
0 \\
0 \\
0 \\
\vdots \\
0 \\
1
\end{array}\right), C=\left(\begin{array}{c}
1 \\
0 \\
0 \\
\vdots \\
0 \\
0
\end{array}\right)^{T}
$$

In theorem 4 we have shown how to satisfy the assumptions of theorem 3 using state feedback. In this section we want to show how to satisfy the assumptions of theorem 3 using measurement feedback. The main result is the following, which is the corresponding result of theorem 4 for measurement feedback.

Theorem 5 Let $j, i \in J$ and $T_{j} \subset R_{j} \subset \mathbb{R}^{2 n}$ be compact sets, $\left\{S_{j}(k) \subset \mathbb{R}^{2 n}, k \in \mathbb{N}\right\}$ a sequence of open sets such that $S_{j}(k) \subset T_{j}$ for large $k$, with $S(k)=\cup_{j=1}^{N} S_{j}(k)$, and $\alpha_{j} \in[0,1)$. Therefore, there exists $l^{*}, k^{*} \in \mathbb{I}$, continuous $\Delta, a_{j}: \mathbb{N} \rightarrow \mathbb{R}^{+}$, positive definite $V_{j}: \mathbb{R}^{2 n} \rightarrow \mathbb{R}^{\geq}$, $j \in J$, and a family of measurement feedback controllers $\left\{C_{j}(k), k \in \mathbb{N}\right\}, j \in J$, such that, if for all $j_{l}, j_{l+1} \in J$ and $k \geq k^{*}$

$\mathbf{E}\left\{\tau\left(t_{j_{l+1}}\right)-\tau\left(t_{j_{l}}\right) \mid \Lambda_{j_{l}}(k)\right\} \geq \Delta(k)$

with $\Lambda_{j_{l}}(k)$ and $\tau_{\mathbb{R}^{n} \backslash S(k)}(t)$ defined as in theorem 3 with $z=(x, \sigma)$, then for all $k \geq k^{*}$ (42) is $\left(\cup_{l=1}^{N} R_{l}, \cup_{l=1}^{N} T_{l}, 1-\right.$ $\left.\Pi_{i=1}^{l^{*}}\left(1-\alpha_{i}\right)\right)-S S$ with measurement feedback switching controllers $\{C(k), k \in \mathbb{I N}\}=\left\{C_{r(t)}(k), k \in \mathbb{N}\right\}$.

Proof. We prove that the assumptions of theorem 3 are met. In particular, taking also into account the results of theorems 2 and 3 , this happens if for some $k^{*} \in \mathbb{N}$ and for all $j=1, \ldots, N$ and $k \geq k^{*}$ :

- there exist $P_{m j}(k) \in S P^{n}, R_{m j}(k)>0$, continuous positive definite $q_{j}: \mathbb{R}^{\geq} \rightarrow \mathbb{R}^{\geq}$and $C^{1}$ functions $\delta_{j}$ : $\mathbb{R}^{\geq} \rightarrow(0,1], j=1, \ldots, N$, such that $\nabla_{s} \delta_{j}(s) \leq \delta_{j}(s)$ for all $s \geq 0$ and

$$
\begin{aligned}
& e^{T}\left[P_{m j}(k) A_{j}+A_{j}^{T} P_{m j}(k)\right. \\
& +h_{j}^{2}(x) P_{m j}(k) B_{j} B_{j}^{T} P_{m j}(k)-R_{m j}^{-1}(k) C_{j}^{T} C_{j} \\
& \left.+P_{s f j}(k) B_{j} R_{s f j}^{-1}(k) B_{j}^{T} P_{s f j}(k)\right] e
\end{aligned}
$$




$$
+\sum_{i=1}^{N} \gamma_{j i} \psi_{i}\left(\|e\|_{P_{m i}(k)}\right) / \delta_{i}\left(\|e\|_{P_{m i}(k)}\right) \leq-q_{j}\left(\|e\|_{P_{m i}(k)}\right)
$$

for all $x \in \Omega_{s f j}(k):=\left\{x \in \mathbb{R}^{n}:(1 / 2)\|x\|_{P_{s f j}(k)}^{2}\right\}$, with $\psi_{j}(s)=\int_{0}^{s} \delta_{j}(s) d s$ and $P_{s f j}(k)$ is defined as in theorem 4 ,

- there exist a continuous locally Lipschitz function $\eta_{j}$ : $\mathbb{R}^{m} \rightarrow \mathbb{R}^{m}$ such that

$$
\frac{\sup _{(x, \sigma) \in R_{j}}\left[\|x\|_{P_{s f j}(k)}^{2}+\psi_{j}\left(\|e\|_{P_{m j}(k)}^{2}\right)\right]}{k} \leq \alpha_{j}
$$

and

$$
\begin{aligned}
& \left\|\eta_{j}\left(-F_{j}(k)(x-e)\right)+F_{j}(k) x\right\|_{R_{s f j}(k)}^{2} \\
& -\frac{\|x\|_{Q_{s f j}(k)}^{2}}{2}+h_{j}^{2}(x) B_{j}^{T} P_{m j}(k) B_{j} \leq \delta_{j}\left(\|e\|_{P_{m j}(k)}^{2}\right) . \\
& \cdot\left[\left\|F_{j}(k) e\right\|_{R_{s f j}(k)}^{2}+\frac{1}{2} q_{j}\left(\|x-\sigma\|_{P_{m j}(k)}^{2}\right)\right]
\end{aligned}
$$

for all $x \in \Omega_{s f j}(k)$ and $e \in \mathbb{R}^{n}$, with $F_{j}(k)=$ $-R_{s f j}^{-1}(k) B_{j}^{T} P_{s f j}(k)$ and $Q_{s f j}(k)=\nu_{s f j}\|x\|_{P_{j}(k)}^{2}+$ $\sum_{i=1}^{N} \gamma_{j i}\|x\|_{P_{i}(k)}^{2}$.

- there exist $\varphi_{j i}: \mathbb{N} \rightarrow \mathbb{R}^{+}$such that for all $j_{l}, j_{l+1} \in J$

$$
\Omega_{j_{l+1}}(k) \cap \Omega_{j_{l}}(k) \supset \Omega_{j_{l}}\left(\varphi_{j_{l} j_{l+1}}(k)\right)
$$

and there exists $l^{*} \in \mathbb{N}$ such that for all $j_{1}, \ldots, j_{l^{*}} \in J$

$$
S(k) \subset \Omega_{j_{1}}\left(\Phi_{j_{l^{*}}}(k)\right) \subset T
$$

where $\Omega_{j}(k):=\left\{z \in \mathbb{R}^{2 n}:\|x\|_{P_{s f j}(k)}^{2}+\psi_{j}(\| x-\right.$ $\left.\sigma \|_{P_{m_{j}}(k)}^{2} \leq k\right\}$ and $\Phi_{j}(k)=\varphi_{j_{l} j_{l+1}} \circ \cdots \varphi_{j_{2} j_{3}} \circ \varphi_{j_{1} j_{2}}(k)$, $\Phi_{0}(k)=k$ and $\varphi_{j j}(k)=k$.

We will use similar arguments to those of [1] for choosing $\eta_{j}$ and $\delta_{j}$ in such a way to satisfy (49)-(51). However, in the presence of a Markov chain we have in (49) the additional term $\sum_{i=1}^{N} \gamma_{j i} \psi_{i}\left(\|e\|_{P_{m i}(k)}\right) / \delta_{i}\left(\|e\|_{P_{m i}(k)}\right)$ for which the choices of $\eta_{j}$ and $\delta_{j}$ pointed out in [1] are not satisfactory any more. Thus, we have to prove that there exists some other choice of these functions satisfying (49)-(51). For, let $r \geq 4(n-1)+1$ and

$\delta_{j}(s)=\frac{(1+s)^{\frac{1-r}{r}}}{\zeta(k) r}, \psi_{j}(s)=\frac{(1+s)^{\frac{1}{r}}-1}{\zeta(k)}$

where $\zeta: \mathbb{R}^{+} \rightarrow \mathbb{R}^{+}$is some $C^{0}$ function (to be specified later) such that $\lim _{k \rightarrow \infty} \zeta(k)=\infty$. Note that, since $r \geq 1$,

$\nabla_{s} \delta_{j}(s)=\frac{1-r}{\zeta(k) r^{2}}(1+s)^{\frac{1-2 r}{r}} \leq 0 \leq \delta_{j}(s), \forall s \geq 0$
Moreover,

$\psi_{i}(s) / \delta_{i}(s)=r\left[1+s-(1+s)^{\frac{r-1}{r}}\right] \leq r s, \forall s \geq 0$

Thus, (49) is solvable for some $P_{m j}(k) \in S P^{n}$ with $q_{j}(s)=\zeta^{2}(k) s$ if

$$
\begin{aligned}
& P_{m j}(k) A+A^{T} P_{m j}(k)+h_{j}^{2}(x) P_{m j}(k) B B^{T} P_{m j}(k) \\
& -R_{m j}^{-1}(k) C^{T} C+P_{s f j}(k) B R_{s f j}^{-1}(k) B^{T} P_{s f j}(k) \\
& +r \sum_{i=1}^{N} \gamma_{j i} P_{m i}(k) \leq-\zeta^{2}(k) P_{m j}(k)
\end{aligned}
$$

is solvable for some $P_{m j}(k) \in S P^{n}$. To construct such $P_{m j}(k) \in S P^{n}$, define

$$
\begin{aligned}
P_{m j}^{(n)}(k) & =\left[P_{m j}\right]_{n n}(k) \\
P_{m j}^{(n-1)}(k) & =\left(\begin{array}{cc}
{\left[P_{m j}\right]_{n-1, n-1}(k)} & {\left[P_{m j}\right]_{n-1, n}(k)} \\
{\left[P_{m j}\right]_{n, n-1}(k)} & {\left[P_{m j}\right]_{n n}(k)}
\end{array}\right) \\
P_{m j}^{(i)}(k) & =\left(\begin{array}{cc}
{\left[P_{m j}\right]_{i i}(k)} & v_{j i}(k) \\
v_{j i}^{T}(k) & P_{m j}^{(i+1)}(k)
\end{array}\right), 1 \leq i \leq n-2
\end{aligned}
$$

where $\left[P_{m j}\right]_{l i}(k)=\left[P_{m j}\right]_{i l}(k)$ and $v_{i}(k)=\operatorname{row}\left(\left[P_{m j}\right]_{i, i+1}(k)\right.$, $0, \ldots, 0)$. Moreover, define

$$
\begin{aligned}
Q_{m j}^{(n)}(k)= & 2\left[P_{m j}\right]_{n, n-1}(k)+\omega_{j n}\left(P_{m j}^{(n)}\right) \\
Q_{m j}^{(i)}(k)= & \left(\begin{array}{cc}
2\left[P_{m j}\right]_{i, i-1}(k)+\omega_{j i}\left(P_{m j}^{(i)}\right) & \xi_{j i}^{T}\left(P_{m j}^{(i)}\right) \\
\xi_{j i}\left(P_{m j}^{(i)}\right) & Q_{m j}^{(i+1)}(k)
\end{array}\right), \\
i=2, \ldots, n-1 & \\
Q_{m j}^{(1)}(k) & =\left(\begin{array}{cc}
-L_{j}(k)+\omega_{j 1}\left(P_{m j}^{(1)}(k)\right) & \xi_{j 1}^{T}\left(P_{m j}^{(1)}(k)\right) \\
\xi_{j 1}\left(P_{m j}^{(1)}(k)\right) & Q_{m j}^{(2)}(k)
\end{array}\right)(58)
\end{aligned}
$$

where $L_{j}: \mathbb{R}^{+} \rightarrow \mathbb{R}^{+}$is a $C^{0}$ function and $\omega_{j i}\left(P_{m j}^{(i)}\right)$ and $\xi_{j i}\left(P_{m j}^{(i)}\right)$ are linear functions of the entries of $P_{m j}^{(i)}$. By direct calculations

$$
\begin{aligned}
& Q_{m j}^{(1)}(k)=P_{m j}^{(1)}(k)(A+I)+(A+I)^{T} P_{m j}^{(1)}(k) \\
& -L_{j}(k) C^{T} C
\end{aligned}
$$

We claim that for each $\left[P_{m j}\right]_{n n}(k)>0$ such that

$$
\frac{\|x\|_{Q_{s f j}(k)}^{2}}{8} \geq\left[P_{m j}\right]_{n n}(k) h_{j}^{2}(x)=h_{j}^{2}(x) B^{T} P_{m j}(k) B(60)
$$

for all $x \in \Omega_{s f j}(k)$, there exist $C^{0}$ functions $L_{j}^{*}: \mathbb{R}^{+} \rightarrow$ $\mathbb{R}^{+}$and $P_{m j}^{(1)}: \mathbb{R}^{+} \rightarrow S P^{n}$ such that $Q_{m j}^{(1)}(k) \in S N^{n}$ for all $C^{0}$ functions $L_{j}(k) \geq L_{j}^{*}(k)$. Indeed,

- pick $\left[P_{m j}\right]_{n, n-1}<0$ such that $Q_{m j}^{(n)}<0$ and $\left[P_{m j}\right]_{n-1, n-1}>0$ such that $P_{m j}^{(n-1)} \in S P^{2}$. 
- Pick $\left[P_{m j}\right]_{n-1, n-2}(k)<0$ such that $Q_{m j}^{(n-1)}(k) \in S N^{2}$ and $\left[P_{m j}\right]_{n-2, n-2}(k)>0$ such that $P_{m j}^{(n-2)}(k) \in S P^{3}$.

- At step $i$, fix $\left[P_{m j}\right]_{n-i, n-1-i}(k)<0$ such that $Q_{m j}^{(n-i)}(k) \in S N^{i+1}$ and $\left[P_{m j}\right]_{n-1-i, n-1-i}(k)>0$ such that $P_{m j}^{(n-1-i)}(k) \in S P^{i+2}$.

- Finally, fix $\left[P_{m j}\right]_{11}(k)>0$ such that $P_{m j}^{(1)}(k) \in S P^{n}$ and $L_{j}^{*}(k)>0$ such that $Q_{m j}^{(1)}(k) \in S N^{n}$ for all $C^{0}$ functions $L_{j}(k) \geq L_{j}^{*}(k)$.

Define

$$
\begin{aligned}
P_{m j}(k) & =\widetilde{P}_{m j}(\zeta(k))=Z(\zeta(k)) P_{m j}^{(1)}(k) Z(\zeta(k)), \\
Z(\zeta(k)) & =\operatorname{diag}\left\{\zeta^{2(n-1)}(k), \zeta^{2(n-2)}(k), \ldots, 1\right\}
\end{aligned}
$$

In what follows, for sake of simplicity we will omit the argument $k$ when there is no ambiguity. We claim that there exists a $C^{0}$ function $\zeta_{j 1}^{*}: \mathbb{R}^{+} \rightarrow \mathbb{R}^{+}$ such that $P_{m j}$, defined as in (61), solves (49) with $R_{m j} \in\left(0,1 /\left[L_{j} \zeta^{2(2 n-1)}\right]\right)$ for all $C^{0}$ functions $\zeta \geq \zeta_{j 1}^{*}$. Indeed, substituting in (49), left and right-multiplying by $Z^{-1}(\zeta(k))$ and dividing both members by $\zeta^{2}(k)$, we obtain

$$
\begin{aligned}
& P_{m j}^{(1)}\left(A+U_{j 1}(\zeta)\right)+\left(A+U_{j 1}(\zeta)\right)^{T} P_{m j}^{(1)} \\
& -\frac{R_{m j}^{-1} C_{j}^{T} C_{j}}{\zeta^{2(2 n-1)}}+U_{j 2}(\zeta) \leq-P_{m j}^{(1)}
\end{aligned}
$$

where $U_{j 1}, U_{j 2}: \mathbb{R}^{+} \rightarrow \mathbb{R}^{n \times n}$ are $C^{0}$ functions such that $\lim _{\zeta \rightarrow \infty} U_{j i}(\zeta)=0, i=1,2$. Choose $\zeta_{j 1}^{*}: \mathbb{R}^{+} \rightarrow \mathbb{R}^{+}$ such that $\lim _{k \rightarrow \infty} \zeta_{j 1}^{*}(k)=\infty$ and

$$
P_{m j}^{(1)} U_{j 1}(\zeta)+U_{j 1}^{T}(\zeta) P_{m j}^{(1)}+U_{j 2}(\zeta) \leq P_{m j}^{(1)}
$$

for all $C^{0}$ functions $\zeta \geq \zeta_{j 1}^{*}$. By (59) and (63), it follows that (62) (and, thus, (49)) is indeed true.

Next, we satisfy (50). Since for $e \in \mathbb{R}^{n}, r \geq 4(n-1)+1$ and for all $\zeta(k) \geq 1$

$$
\frac{1}{\zeta(k)}\left(\left(1+\|e\|_{\widetilde{P}_{m j}(\zeta(k))}^{2}\right)^{\frac{1}{r}}-1\right) \leq \frac{\|e\|^{2 / r} \lambda_{\max }^{1 / 2}\left(P_{m j}^{(1)}(k)\right.}{\zeta^{1 / 2}(k)}
$$

then there exists a $C^{0}$ function $\zeta_{j 2}^{*}: \mathbb{R}^{+} \rightarrow \mathbb{R}^{+}$such that for all $C^{0}$ functions $\zeta \geq \zeta_{j 2}^{*}$

$$
\begin{aligned}
& \lim _{k \rightarrow \infty} \frac{1}{k}\left[\|x\|_{P_{s f j}(k)}^{2}\right. \\
& \left.+\frac{1}{\zeta(k)}\left(\left(1+\|x-\sigma\|_{\widetilde{P}_{m j}(\zeta(k))}^{2}\right)^{\frac{1}{r}}-1\right)\right]=0
\end{aligned}
$$

for each $(x, \sigma) \in \Omega_{s f j}(k) \times \mathbb{R}^{n}$, which proves (50).
Now, we prove (51). Let

$$
\begin{aligned}
& F_{j}(k)=R_{s f j}^{-1}(k) \operatorname{row}(0,0, \ldots, 0,1) P_{s f j}(k) \\
& \eta_{j}(s)=\operatorname{col}\left(\eta_{j 1}(s), \ldots, \eta_{j m}(s)\right), s=\operatorname{col}\left(s_{1}, \ldots, s_{m}\right), \\
& \eta_{j i}\left(s_{i}\right)=\frac{s_{i}}{\left(1+\max \left\{0,\left|s_{i}\right|-\max _{x \in \Omega_{s f j}}\left|F_{j} x\right|\right\}\right)^{\frac{r-1}{r}}}(6)
\end{aligned}
$$

Taking into account (60), condition (51) is satisfied for all $x \in \Omega_{s f j}(k)$ and $e \in \mathbb{R}^{n}$ if there exists a $C^{0}$ function $\zeta_{j 3}^{*}: \mathbb{R}^{+} \rightarrow \mathbb{R}^{+}$such that

$$
\begin{aligned}
& \frac{\zeta^{2}\|e\|_{\widetilde{P}_{m j}(\zeta)}^{2}+\left\|F_{j} e\right\|_{R_{s f j}}^{2}}{r \zeta\left(1+\|e\|_{\widetilde{P}_{m j}(\zeta)}^{2}\right)^{\frac{r-1}{r}}}+\frac{1}{4}\|x\|_{Q_{s f j}}^{2} \\
& -\| F_{j} x-\eta_{j}\left(F_{j}(x-e) \|_{R_{s f j}}^{2} \geq 0\right.
\end{aligned}
$$

for all $C^{0}$ functions $\zeta \geq \zeta_{j 3}^{*}, x \in \Omega_{s f j}(k)$ and $e \in \mathbb{R}^{n}$. In order to prove (66), find a covering $\cup_{i=1}^{3} M_{j i}$ of $\{(x, e) \in$ $\left.\Omega_{s f j}(k) \times \mathbb{R}^{n}\right\}$, with

$$
\begin{aligned}
M_{j 1}= & \left\{(x, e) \in \Omega_{s f j}(k) \times \mathbb{R}^{n}:\|x-e\| \leq \vartheta_{j 1} ;\right. \\
& \left.\|e\| \leq \frac{\vartheta_{j 1}}{2}\right\}, \vartheta_{j 1}>0 \\
M_{j 2}= & \left\{(x, e) \in \Omega_{s f j}(k) \times \mathbb{R}^{n}:\|x-e\| \geq \vartheta_{j 1} ;\right. \\
& \left.\|e\| \leq \vartheta_{j 2}\right\}, \vartheta_{j 2} \leq \frac{\vartheta_{j 1}}{2} \\
M_{j 3}= & \left\{(x, e) \in \Omega_{s f j}(k) \times \mathbb{R}^{n}:\|e\| \geq \vartheta_{j 2}\right\}
\end{aligned}
$$

for some $\vartheta_{j 2}>0$ and $\vartheta_{j 1}>0$ such that $\eta_{j}\left(-F_{j}(x-e)\right)=$ $-F_{j}(x-e)$ for all $\|x-e\| \leq \vartheta_{j 1}$.

First of all, it is easy to see that there exists a $C^{0}$ function $\zeta_{j 4}^{*}: \mathbb{R}^{+} \rightarrow \mathbb{R}^{+}$such that (66) holds for all $C^{0}$ functions $\zeta \geq \zeta_{j 4}^{*}$ and $(x, e) \in M_{j 1}$.

Moreover, (66) holds for all $(x, e) \in M_{j 2}$ for some $\vartheta_{j 2} \leq$ $\frac{\vartheta_{j 1}}{2}$ and for all $k>0$. Indeed, for all $(x, e) \in M_{2}$, since $\vartheta_{1}>\vartheta_{2}$ we have $\frac{1}{4}\|x\|_{Q_{s f j}}^{2}>0$. It follows that for any such a $x$ by continuity there exists $e_{j x}>0$ such that (66) holds for all $\|e\| \leq e_{j x}$ and for all $C^{0}$ functions $\zeta \geq \zeta_{j 4}^{*}$. Since $N_{j}=\left\{x \in \Omega_{s f j}(k):\|x\| \geq \frac{\vartheta_{j 1}}{2}\right\}$ is compact and $\vartheta_{j 1}>0$, one can take $\vartheta_{j 2}=\min \left\{\frac{\vartheta_{j 1}}{2}, \min _{x \in N_{j}} e_{j x}\right\}$.

We are left with proving that there exists a $C^{0}$ function $\zeta_{j 5}^{*}: \mathbb{R}^{+} \rightarrow \mathbb{R}^{+}$such that (66) holds for all $(x, e) \in M_{j 3}$ and for all $C^{0}$ functions $\zeta \geq \zeta_{j 5}^{*}$. Since $\|e\|_{\widetilde{P}_{m j}(\zeta(k))}^{2} \geq$ $\|e\|^{2} \lambda_{m i n}\left(P_{m j}^{(1)}(k)\right)$ we have

$$
\frac{\zeta^{2}\|e\|_{\widetilde{P}_{m j}(\zeta)}^{2}+\left\|F_{j} e\right\|_{R_{s f j}}^{2}}{r \zeta\left(1+\|e\|_{\widetilde{P}_{m j}(\zeta)}^{2}\right)^{\frac{r-1}{r}}}+\frac{1}{4}\|x\|_{Q_{s f j}}^{2}
$$




$$
\begin{aligned}
& -\| F_{j} x-\eta_{j}\left(F_{j}(x-e) \|_{R_{s f j}}^{2} \geq \frac{\zeta \lambda_{\min }\left(P_{m j}^{(1)}\right)\|e\|^{2}}{r\left(1+\lambda_{\min }\left(P_{m j}^{(1)}\right)\|e\|^{2}\right)^{\frac{r-1}{r}}}\right. \\
& -\sum_{i=1}^{m} 4\left[2 \max _{x \in \Omega_{s f j}}\left(\left[F_{j}\right]_{i} x\right)^{2}\right. \\
& +\frac{\max ^{2}\left\{0,\left|\left[F_{j}\right]_{i}(x-e)\right|-\max _{x \in \Omega_{s f j}}\left|\left[F_{j}\right]_{i} x\right|\right\}}{\left.\left(1+\max ^{2}\left\{0,\left|\left[F_{j}\right]_{i}(x-e)\right|-\max _{x \in \Omega_{s f j}}\left|\left[F_{j}\right]_{i} x\right|\right\}\right)^{\frac{r-1}{r}}\right] R_{s f j}} \\
& \geq \frac{\zeta \lambda_{\min }\left(P_{m j}^{(1)}\right)\|e\|^{2}}{r\left(1+\lambda_{\min }\left(P_{m j}^{(1)}\right)\|e\|^{2}\right)^{\frac{r-1}{r}}}-\sum_{i=1}^{m} 4\left[3 \max _{x \in \Omega_{s f j}}\left(\left[F_{j}\right]_{i} x\right)^{2}\right. \\
& +\frac{\left(\left[F_{j}\right]_{i}(x-e)\right)^{2}}{\left.\left(1+\left(\left[F_{j}\right]_{i}(x-e)\right)^{2}\right)^{\frac{r-1}{r}}\right]} R_{s f j} \geq \\
& \frac{\zeta \lambda_{\min }\left(P_{m j}^{(1)}\right)\|e\|^{2}}{r\left(1+\lambda_{\min }\left(P_{m j}^{(1)}\right)\|e\|^{2}\right)^{\frac{r-1}{r}}}-\sum_{i=1}^{m} 4\left[5 \max _{x \in \Omega_{s f j}}\left\|\left[F_{j}\right]_{i}\right\|^{2}\|x\|^{2}\right. \\
& \left.+2 \frac{\left\|\left[F_{j}\right]_{i}\right\|^{2}\|e\|^{2}}{\left(1+\left\|\left[F_{j}\right]_{i}\right\|^{2}\|e\|^{2}\right)^{\frac{r-1}{r}}}\right] R_{s f j}
\end{aligned}
$$

This implies the existence of a $C^{0}$ function $\zeta_{j 5}^{*}: \mathbb{R}^{+} \rightarrow$ $\mathbb{R}^{+}$such that (66) holds for all $(x, e) \in M_{j 3}$ and for all $C^{0}$ functions $\zeta \geq \zeta_{j 5}^{*}$. Pick $\zeta_{j 3}^{*} \geq \max \left\{\zeta_{j 4}^{*}, \zeta_{j 5}^{*}\right\}$. Finally, we conclude that (49)-(51) hold as long as $\zeta: \mathbb{R}^{+} \rightarrow \mathbb{R}^{+}$is any $C^{0}$ function such that $\zeta \geq \max _{j=1, \ldots, N}\left\{\zeta_{j 1}^{*}, \zeta_{j 2}^{*}, \zeta_{j 3}^{*}\right\}$.

Finally, (52)-(53) can be proved as in theorem 4, once we show that for a proper choice of $\zeta(k), a_{j}(k)>0$ and $b_{j}(k) \in(0,1 / 2]$ we have $\left\{z \in \mathbb{R}^{2 n}: V_{j}(z) \leq a_{j}(k)\right\} \subset$ $S_{j}(k)$ and the functions $I_{j}(s):=\int_{a_{j}(k)}^{s}\left(1 / \nu_{j}(s)\right) d s$, with $\nu_{j}(s):=a_{j}(k) b_{j}(k) s$ if $|s|>a_{j}(k)$ and $=b_{j}(k) s^{2}$ otherwise, are such that

$$
\begin{aligned}
& \nu_{j}\left(V_{j}(z)\right)+\sum_{l=1}^{N} V_{l}(z) \gamma_{j l} \\
& \leq\|x\|_{Q_{s f j}(k)}^{2}+\delta_{j}\left(\|x-\sigma\|_{P_{m j}(k)}^{2}\right) q_{j}\left(\|x-\sigma\|_{P_{m j}(k)}^{2}\right) \\
& +\sum_{l=1}^{N} \psi_{l}\left(\|e\|_{P_{m j}(k)}^{2}\right) \gamma_{j l}
\end{aligned}
$$

with $z=(x, \sigma), V_{j}(z)=\|x\|_{P_{s f j}}^{2}+\psi_{j}\left(\|e\|_{P_{m j}}\right.$ and for all $z \in \Omega_{j}(k)$. Indeed, let $\nu_{s f j}$ and $\Omega_{s f j}$ be as in the proof of theorem 4 . Since $\zeta(k) \geq 1, k \geq 1$ and $x \in \Omega_{s f j}(k)$, there exists $m_{j}>0$ such that $(1+s)^{1 / r}-1 \leq m_{j} s /(1+s)$ for all $s \in[0,1]$, then for $k \geq V_{j}(z) \geq a_{j}(k),\|x-\sigma\|_{P_{m j}(k)}^{2} \leq 1$, $\zeta(k) \geq \max \left\{1, m_{j} r\right\}$ and $2 b_{j}(k) k \leq \nu_{s f j}$

$$
\begin{aligned}
& \nu_{j}\left(V_{j}(z)\right)=b_{j}(k)\left(V_{j}(z)\right)^{2} \leq 2 b_{j}(k)\left\{k\|x\|_{P_{s f j}(k)}^{2}\right. \\
& \left.+\left[\left(1+\|x-\sigma\|_{P_{m j}(k)}^{2}\right)^{1 / r}-1\right]^{2}\right\} \leq \nu_{s f j}\|x\|_{P_{s f j}(k)}^{2}
\end{aligned}
$$

$$
\begin{aligned}
& \left.+m_{j}\left[\left(1+\|x-\sigma\|_{P_{m j}(k)}^{2}\right)^{1 / r}-1\right]\right\} \frac{\|x-\sigma\|_{P_{m j}(k)}^{2}}{1+\|x-\sigma\|_{P_{m j}(k)}^{2}} \\
& \leq \nu_{s f j}\|x\|_{P_{s f j}(k)}^{2}+(\zeta(k) / r)\left(1+\|x-\sigma\|_{P_{m j}}^{2}\right)^{1 / r} \\
& \cdot \frac{\|x-\sigma\|_{P_{m j}(k)}^{2}}{1+\|x-\sigma\|_{P_{m j}(k)}^{2}}=\nu_{s f j}\|x\|_{P_{s f j}(k)}^{2} \\
& +\delta_{j}\left(\|x-\sigma\|_{P_{m j}(k)}^{2}\right) q_{j}\left(\|x-\sigma\|_{P_{m j}(k)}^{2}\right)
\end{aligned}
$$

On the other hand, for $k \geq V_{j}(z) \geq a_{j}(k)$, $\| x-$ $\sigma \|_{P_{m j}(k)}^{2} \geq 1, \zeta(k) \geq \max \{1, \sqrt{2 k r}\}$ and $2 b_{j}(k) k \leq \nu_{s f j}$ we have

$$
\begin{aligned}
& \nu_{j}\left(V_{j}(z)\right) \leq b_{j}(k)\left(V_{j}(z)\right)^{2} \leq 2 b(k)\left\{k\|x\|_{P_{s f j}(k)}^{2}\right. \\
& \left.+\left(1 / \zeta^{2}(k)\right)\left[\left(1+\|x-\sigma\|_{P_{m j}(k)}^{2}\right)^{1 / r}-1\right]^{2}\right\} \\
& \leq \nu_{s f j}(k)\|x\|_{P_{s f j}(k)}^{2}+(k / \zeta(k))\left[\left(1+\|x-\sigma\|_{P_{m j}(k)}^{2}\right)^{1 / r}\right. \\
& -1] \leq \nu_{s f j}\|x\|_{P_{s f j}(k)}^{2}+(\zeta(k) / r)\left(1+\|x-\sigma\|_{P_{m j}}^{2}\right)^{1 / r} . \\
& \quad \frac{\|x-\sigma\|_{P_{m j}(k)}^{2}}{1+\|x-\sigma\|_{P_{m j}(k)}^{2}}=\nu_{s f j}(k)\|x\|_{P_{s f j}(k)}^{2} \\
& \left.+\delta_{j}\left(\|x-\sigma\|_{P_{m j}(k)}^{2}\right) q_{j}\|x-\sigma\|_{P_{m j}(k)}^{2}\right)
\end{aligned}
$$

This with (70) proves (69) for $k \geq V_{j}(z) \geq a_{j}(k)$. When $0 \leq V_{j}(z) \leq a_{j}(k)$ then $\nu_{j}\left(V_{j}(z)\right)=b_{j}(k)\left(V_{j}(z)\right)^{2}$ and we can prove (69) as above in the case $k \geq V_{j}(z) \geq a_{j}(k)$.

According to theorems 2 and $3,(42)$ is $\left(\cup_{l=1}^{N} R_{l}, \cup_{l=1}^{N} T_{l}, 1-\right.$ $\left.\prod_{i=1}^{l^{*}}\left(1-\alpha_{i}\right)\right)$-SS with switching controller $\{C(k), k \in$ $\mathbb{N}\}=\left\{C_{r(t)}(k), k \in \mathbb{N}\right\}$ where $C_{j}(k)$ :

$$
\begin{aligned}
& u=-\eta_{j}\left[F_{j}(k) \sigma\right] \\
& d \sigma=\left[\left(A-P_{m j}^{-1}(k) C^{T} R_{m j}^{-1}(k) C\right) \sigma+B u\right] d t \\
& +P_{m j}^{-1}(k) C^{T} R_{m j}^{-1}(k) d y
\end{aligned}
$$

where $F_{j}(k)$ and $\eta_{j}(s)$ are defined as in (65). .

\section{Conclusions}

We have introduced a notion of stabilization for switching systems. Peculiar to this definition is the fact to evaluate the probability of the trajectory to pass through a sequence of decreasing sets $D_{j}, j=1, \ldots$, , at each switching and approaching the target. Our main contribution is to prove that if the expectation of the dwelltime, conditionally to the event that the trajectory goes from $D_{j}$ to $D_{j+1}$, is "sufficiently large" then the switching system is stable under switching with guaranteed probability (theorem 3). As an application of our design tool, we consider a significant class of nonlinear stochastic systems and show in detail how to construct both a state feedback and a measurement feedback dwell-time controller. Our approach is promising for applications to more general class of nonlinear stochastic systems. 


\section{References}

[1] S. Battilotti, A. De Santis, Stabilization in probability of nonlinear stochastic systems with guaranteed region of attraction, IEEE Transactions on Automatic Control, 48, pp. 1-15, 2003.

[2] S. Battilotti, A. De Santis, Robust output feedback control of nonlinear stochastic systems using neural networks, IEEE Transactions on Neural Networks, 14, pp. 103-116, 2003.

[3] S. Battilotti, A unifying framework for the semiglobal stabilization of nonlinear uncertain systems via measurement feedback, IEEE Transactions on Automatic Control, 46, 3$16,2001$.

[4] A.R. Teel, L. Praly, Tools for semiglobal stabilization by partial state and output feedback, SIAM Journ. Contr. Optim., 33, 1995, 1443-1488.

[5] R. Z. Khas'minskii, Stochastic stability of differential equations, Rockville, Sjithoff \& Noordhoff, 1980.

[6] R.S. Lipster, A.N. Shiryayev, Statistics of random processes I: general theory, Springer Verlag, Berlin, 1977.

[7] S. Morse, Supervisory control of families of linear set-point controllers - part I: exact matching, IEEE Transactions on Automatic Control, 41, pp. 1413-1431, 1996.

[8] C. De Persis, R. De Santis, S. Morse, Supervisory control with state-dependent dwell-time logic and constraints, Automatica, 40, 269-275, 2004.

[9] D. Angeli, E. Mosca, Lyapunov based switching supervisory control of nonlinear uncertain systems, IEEE Transactions on Automatic Control, 47, pp. 500-505, 2002.

[10] X. Mao, Exponential stability of stochastic delay interval system with Markovian switching, IEEE Transactions on Automatic Control, 47, pp. 1604-1612, 2002.

[11] M.S. Branicky, Multiple Lyapunov functions and other analysis tools for switched and hybrid systems, IEEE Transactions on Automatic Control, 43, pp. 475-482, 1998.

[12] A. Saberi, Z. Lin, A. Teel, Control of Linear systems with saturating actuators, IEEE Trans. Autom. Contr., 41, 1996 , $368-378$. 